\title{
Fine structure constant and the CMB damping scale
}

\author{
Eloisa Menegoni, ${ }^{1}$ Maria Archidiacono, ${ }^{2}$ Erminia Calabrese, ${ }^{3}$ Silvia Galli, ${ }^{4}$ \\ C. J. A. P. Martins, ${ }^{5}$ and Alessandro Melchiorri ${ }^{2}$ \\ ${ }^{1}$ I.C.R.A. and INFN, Università di Roma "La Sapienza", Ple Aldo Moro 2, 00185, Rome, Italy \\ ${ }^{2}$ Physics Department and INFN, Università di Roma "La Sapienza", Ple Aldo Moro 2, 00185, Rome, Italy \\ ${ }^{3}$ Subdepartment of Astrophysics, University of Oxford, Keble Road, Oxford, OX1 3RH, UK \\ ${ }^{4}$ Institut d'Astrophysique de Paris, UMR 7095-CNRS Paris, Université Pierre et Marie Curie, \\ Boulevard Arago 98bis, 75014, Paris, France \\ ${ }^{5}$ Centro de Astrofísica, Universidade do Porto, Rua das Estrelas, 4150-762 Porto, Portugal
}

(Received 8 February 2012; published 3 May 2012)

\begin{abstract}
The recent measurements of the Cosmic Microwave Background anisotropies at arc minute angular scales performed by the ACT and SPT experiments are probing the damping regime of CMB fluctuations. The analysis of these data sets unexpectedly suggests that the effective number of relativistic degrees of freedom is larger than the standard value of $N_{\text {eff }}=3.04$, and inconsistent with it at more than two standard deviations. In this paper we study the role of a mechanism that could affect the shape of the CMB angular fluctuations at those scales, namely, a change in the recombination process through variations in the fine structure constant. We show that the new CMB data significantly improve the previous constraints on variations of $\alpha$, with $\alpha / \alpha_{0}=0.984 \pm 0.005$, i.e. hinting also at a more than two standard deviation from the current, local, value $\alpha_{0}$. A significant degeneracy is present between $\alpha$ and $N_{\text {eff }}$, and when variations in the latter are allowed the constraints on $\alpha$ are relaxed and again consistent with the standard value. Deviations of either parameter from their standard values would imply the presence of new, currently unknown physics.
\end{abstract}

DOI: 10.1103/PhysRevD.85.107301

PACS numbers: 98.70.Vc, 95.85.Sz, 98.80.Es

\section{INTRODUCTION}

The recent observations from Cosmic Microwave Background (CMB hereafter) satellite, balloon-borne, and ground-based experiments ([1-4]), galaxy redshift surveys [5], and luminosity distance measurements, have fully confirmed the theoretical predictions of the standard $\Lambda \mathrm{CDM}$ cosmological model. This not only allows stringent constraints on the parameters of the model but can be fruitfully used to constrain nonstandard physics at the fundamental level, such as classes of elementary particle models predicting a different radiation content in the Universe.

In this respect, an interesting discrepancy with the expectations of the standard model has recently been uncovered in the small CMB scale measurements of the ACT [2] and SPT [4] experiments. Namely, the effective number of relativistic degrees of freedom $N_{\text {eff }}$ (see e.g. [6] for a definition) has been reported as higher (at more than two standard deviations) than the expected standard value of $N_{\text {eff }}=3.046 .{ }^{1}$ This result has been confirmed by several recent analyses of the ACT and SPT datasets (see e.g. [8-12]).

While a confirmation from future measurements is clearly needed, the current preference for $N_{\text {eff }} \sim 4$ is

\footnotetext{
${ }^{1}$ This is the value expected in the case of 3 relativistic neutrinos species. The little deviation from $N_{\text {eff }}=3$ takes into account effects from the noninstantaneous neutrino decoupling from the primordial photon-baryon plasma (see e.g. [7]).
}

stimulating a growing interest since it could be explained in different ways, including an extra relativistic particle at decoupling (as light axions or sterile neutrinos), extra dimensions, or early dark energy. (see e.g. [8] and references therein).

However it is important to stress that the current bounds on $N_{\text {eff }}$ rely on the assumption of a theoretical model. More recently the dependence of the constraints on $N_{\text {eff }}$ on the assumption of a flat universe or a different dark energy component have been investigated by several authors ([13-15]). Here we revisit the issue, by obtaining analogous constraints in the framework of a nonstandard recombination process.

As expected (see e.g. [9]) a variation of $N_{\text {eff }}$ affects the value of the Hubble parameter $H$ at recombination. This changes two very important scales in CMB anisotropy physics: the size of the sound horizon and the damping scale at recombination. An approximate expression for the damping scale is given by

$$
r_{d}^{2}=(2 \pi)^{2} \int_{0}^{a_{*}} \frac{d a}{a^{3} \sigma_{T} n_{e} H}\left[\frac{R^{2}+\frac{16}{15}(1+R)}{6\left(1+R^{2}\right)}\right],
$$

where $n_{e}$ is the number density of free electrons, $\sigma_{T}$ is the Thomson cross section, $a_{*}$ is the scale factor at recombination, and $R=3 \rho_{b} /\left(4 \rho_{\gamma}\right)$ is proportional to the ratio between the baryon and photon densities. It is clear that a change in $H$ could be compensated by a change in $n_{e}$ and $a_{*}$ in order to keep the same damping scale. Consequently, a change in the recombination process, motivated by some 
nonstandard and unaccounted mechanism, could alter the current conclusions on $N_{\text {eff }}$.

Possible changes in the recombination process have been investigated by several authors. Dark matter annihilation, for example, could significantly alter the evolution of the free electron density $n_{e}$ by the injection of extraionizing photons around recombination (see e.g. [16] and references therein). Another possible mechanism, which we consider in this paper, is based on the hypothesis of a change in the fundamental constants of nature, specifically the fine structure constant, $\alpha$.

Changing $\alpha$ modifies the strength of the electromagnetic interaction and therefore modifies the formation of CMB anisotropies by changing the differential optical depth (i.e. the scattering rate) due to Thomson scattering between electrons and photons,

$$
\dot{\tau}=x_{e} n_{e} c \sigma_{T}
$$

where $\sigma_{T}$ is the Thomson cross section, $x_{e}$ is the free electron fraction dependent on the temperature of the electrons and therefore on the scale factor of the universe $a(t)$. The optical depth $\tau$ is then defined as the integral of the scattering rate over time.

These two combined processes change the temperature at last scattering, $T^{*}$, and $x_{e}\left(t_{0}\right)$, the free electron fraction that remains after recombination, both of which influence the CMB anisotropies (see [17]).

The main imprint on CMB power spectrum is a shift in the modulation of the peak heights by baryon drag determined by the relative density of baryons to photons at $\eta^{*}$, $R=3 \rho_{b} /\left(4 \rho_{\gamma}\right) \sim T^{*^{-1}}$.

A variation in $\alpha$ changes the photon diffusion damping length as well and the two effects combined lead to subtle degeneracies between $\Delta \alpha / \alpha$ and $N_{\text {eff }}$.

$\mathrm{CMB}$ anisotropies are therefore one of the canonical ways of constraining variations in the fine structure constant in the early Universe. They provide a measurement of $\alpha$ at the epoch of recombination (see e.g. [18-24]), with a current sensitivity the level of $\sim 1 \%$. In the most recent analysis, parametrizing a variation in the fine structure constant as $\alpha / \alpha_{0}$, where $\alpha_{0}=1 / 137.03599907$ is the standard (local) value and $\alpha$ is the value during the recombination process, the authors of [23] used the $5 \mathrm{yr}$ WMAP data, finding the constraint $0.987 \pm 0.012$ at $68 \%$ c.l. Meanwhile, a recent analysis of a large data set of spectroscopic data from the VLT and Keck telescopes [25] is consistent with earlier claims of variations in the value of $\alpha$ at parts-per-million level at redshifts $z \sim 3$.

In view of this and the recent results from ACT and SPT it is therefore extremely timely to place new bounds on variations of $\alpha$ discussing also the possible degeneracies with $N_{\text {eff }}$. In this paper we indeed perform this kind of analysis, including also possible variations in the abundance of primordial Helium $Y_{p}$ that could similarly change the recombination process. In the next section we describe the analysis method, in Sec. III we present our results, and in Sec. IV we derive our conclusions.

\section{ANALYSIS METHOD}

We perform a COSMOMC [26] analysis combining the following CMB data sets: WMAP7 [1], ACBAR [3], ACT [2], and SPT [4], and we analyze data sets out to $l_{\max }=3000$. We also include information on dark matter clustering from the galaxy power spectrum extracted from the SDSS-DR7 luminous red galaxy sample [5]. Finally, we impose a prior on the Hubble parameter based on the last Hubble Space Telescope observations [27].

The analysis method we adopt is based on the publicly available Monte Carlo Markov Chain package cosmomc [26] with a convergence diagnostic done through the Gelman and Rubin statistic.

We sample the following six-dimensional standard set of cosmological parameters, adopting flat priors on them: the baryon and cold dark matter densities $\Omega_{\mathrm{b}}$ and $\Omega_{\mathrm{c}}$, the Hubble constant $H_{0}$, the optical depth to reionization $\tau$, the scalar spectral index $n_{S}$, and the overall normalization of the spectrum $A_{S}$ at $k=0.002 \mathrm{Mpc}^{-1}$. We consider purely adiabatic initial conditions and we impose spatial flatness. As discussed in the introduction we allow for variations in the fine structure constant $\alpha / \alpha_{0}$ where $\alpha_{0}$ is the current, local, value by modifying the RECFAST recombination subroutine following the procedure described in [23].

We also allow for variations in the effective number of relativistic degrees of freedom $N_{\text {eff }}$ and the primordial Helium abundance $Y_{p}$, otherwise fixed at the values $N_{\text {eff }}=$ 3.046 and $Y_{p}=0.24$, respectively. Since we are varying also the Helium abundance, we considered variations in the fine structure constant also in the process of Helium recombination. $A \sim 5 \%$ change of $\alpha$ for Helium recombination changes the CMB angular spectra by less than $0.5 \%$ up to $\ell=1500$. During reionization the fine structure constant is fixed to the local standard value $\alpha=\alpha_{0}$.

We account for foregrounds contributions including three extra amplitudes: the SZ amplitude $A_{\mathrm{SZ}}$, the amplitude of clustered point sources $A_{C}$, and the amplitude of Poisson distributed point sources $A_{\mathrm{P}}$. We marginalize the contribution from point sources only for the ACT and SPT data, based on the templates provided by [4]. We quote only one joint amplitude parameter for each component (clustered and Poisson distributed). The SZ amplitude is obtained fitting the WMAP data with the WMAP own template, while for SPT and ACT it is calculated using the [28] SZ template at $148 \mathrm{GHz}$; this differs from the analysis performed in [4] where no SZ contribution was considered for the WMAP data.

\section{RESULTS}

As stated in the previous section, we perform three different analyses always considering the same set of 
TABLE I. MCMC estimation of the cosmological parameters from the data set described in the text. Results for the three analyses described in the text are reported. Upper bounds at $95 \%$ c.l. are reported for foregrounds parameters.

\begin{tabular}{lccc}
\hline \hline Parameter & $\alpha / \alpha_{0}$ & $\alpha / \alpha_{0}+N_{\text {eff }}$ & $\alpha / \alpha_{0}+N_{\text {eff }}+Y_{p}$ \\
\hline$\Omega_{b} h^{2}$ & $0.0218 \pm 0.0004$ & $0.0224 \pm 0.0005$ & $0.0223 \pm 0.0007$ \\
$\Omega_{c} h^{2}$ & $0.1144 \pm 0.0034$ & $0.1302 \pm 0.0095$ & $0.1303 \pm 0.0094$ \\
$\tau$ & $0.086 \pm 0.014$ & $0.088 \pm 0.015$ & $0.088 \pm 0.016$ \\
$H_{0}$ & $68.9 \pm 1.4$ & $71.52 \pm 2.0$ & $71.8 \pm 2.1$ \\
$\alpha / \alpha_{0}$ & $0.984 \pm 0.005$ & $0.990 \pm 0.006$ & $0.987 \pm 0.014$ \\
$n_{s}$ & $0.976 \pm 0.013$ & $0.991 \pm 0.015$ & $0.992 \pm 0.016$ \\
$\log \left[10^{10} A_{s}\right]$ & $3.193 \pm 0.037$ & $3.169 \pm 0.040$ & $3.167 \pm 0.042$ \\
$A_{\mathrm{SZ}}$ & $<2.00$ & $<2.00$ & $<2.00$ \\
$A_{C}$ & $<16.0$ & $<15.8$ & $<14.8$ \\
$A_{\mathrm{P}}$ & $<24.7$ & $<24.9$ & $<22.4$ \\
$\Omega_{\Lambda}$ & $0.7137 \pm 0.0070$ & $0.7020 \pm 0.0094$ & $0.704 \pm 0.013$ \\
$\mathrm{Age} / \mathrm{Gyr}$ & $13.76 \pm 0.24$ & $13.18 \pm 0.38$ & $13.15 \pm 0.37$ \\
$\Omega_{m}$ & $0.2863 \pm 0.0070$ & $0.2980 \pm 0.0094$ & $0.296 \pm 0.013$ \\
$\sigma_{8}$ & $0.836 \pm 0.023$ & $0.862 \pm 0.028$ & $0.859 \pm 0.034$ \\
$z_{r e}$ & $10.7 \pm 1.2$ & $11.0 \pm 1.3$ & $11.0 \pm 1.3$ \\
$N_{\mathrm{eff}}$ & - & $4.10_{-0.29}^{+0.24}$ & $4.19_{-0.35}^{+0.31}$ \\
$Y_{p}$ & - & - & $0.215 \pm 0.096$ \\
$\chi_{\min }^{2}$ & 7600.2 & 7596.8 & 7596.5 \\
\hline \hline
\end{tabular}

data but different number of parameters. To the 6 standard $\Lambda$-CDM parameters we cumulatively add as additional free parameters the fine structure constant (first case), the number of relativistic degrees of freedom $N_{\text {eff }}$ (second case), and the primordial Helium abundance $Y_{p}$ (third case). In Table I we report the constraints on the cosmological parameters for these three scenarios.

As we can see the data set considered prefers a value of $\alpha / \alpha_{0}$ smaller than unity at more than two standard deviations when both the $N_{\text {eff }}$ and $Y_{p}$ are kept fixed at their standard values. This result, while interesting, is to be expected since it is clearly driven by data preference for larger values of $N_{\text {eff }}$. Allowing for variations in $N_{\text {eff }}$ significantly shifts the best-fit value for $\alpha / \alpha_{0}$, which is now consistent with the standard value. However, even in this case the best-fit value for $N_{\text {eff }}$ is still $\sim 4$, i.e. allowing for variation in the fine structure constant enlarges the error bars on $N_{\text {eff }}$ by about $\sim 30 \%$ but does not shift the best-fit value towards the standard result. The largest effect on $\alpha$ comes however when also the helium abundance $Y_{p}$ is allowed to vary. In this case, indeed, the errors on $\alpha$ are almost doubled.

We can better understand the impact of $Y_{p}$ on the determination of $\alpha / \alpha_{0}$ by looking at Fig. 1, where we plot the 2-D likelihood contours in the $\alpha / \alpha_{0}-N_{\text {eff }}$ plane in the cases of $Y_{p}=0.24$ and free $Y_{p}$. As we can see when the helium abundance is fixed there is a clear but moderate degeneracy between $\alpha / \alpha_{0}$ and $N_{\text {eff }}$. When $N_{\text {eff }}$ is increased the Hubble parameter at recombination increases. In order to keep the damping scale at the same value fixed by observations [see Eq. (1)] we need to decrease the free electron density at recombination. This can be achieved by simply accelerating the recombination process. This effect is clearly obtained by an increase in the fine structure constant. This explains the direction of the degeneracy in the contour plot.

When also a variation in the Helium abundance is considered, the degeneracy changes direction. A larger value for $Y_{p}$ produces a large free electron fraction at recombination and a smaller value for $N_{\text {eff }}$ is needed to keep the damping scale small. On the other hand a large value for $Y_{p}$ needs large values for $\alpha$. So now small values of $N_{\text {eff }}$ are more compatible with observations when $\alpha$ is larger.

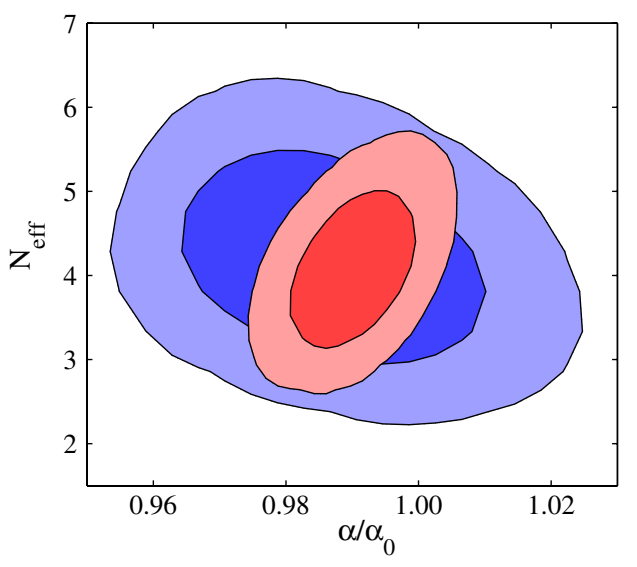

FIG. 1 (color online). Likelihood contour plot for $\alpha / \alpha_{0}$ vs $N_{\text {eff }}$ at $68 \%$ c.l. and $95 \%$ c.l. in the case of $Y_{p}=0.24$ (red smaller contours) and $Y_{p}$ allowed to vary (blue larger contours). 


\section{CONCLUSIONS}

In this paper we have presented new constraints on variations of the fine structure constant from the latest CMB anisotropy measurements of the ACT and SPT experiments, combined with other cosmological data sets. We have found that assuming the standard value for $N_{\text {eff }}$ and a primordial Helium abundance of $Y_{p}=0.24$ the current data favors a lower value for the fine structure constant at more than two standard deviations with $\alpha / \alpha_{0}=0.984 \pm 0.005$.

We have shown that this result relies on the assumption of the number of relativistic degrees of freedom. When we let this parameter vary freely, the standard value is again consistent with the data considered. Varying also the primordial Helium content further enlarges the error bars. Despite the existing degeneracies, the current data offers the tantalizing suggestion of the presence of new physics at the epoch of recombination.

Clearly, further experimental confirmation of the result is needed. Fortunately, the results from the Planck satellite mission, expected to be released early next year, will most probably clarify the issue. The Planck experiment is indeed expected to have a sensitivity of $\Delta N_{\text {eff }} \sim 0.2$ and $\delta\left(\alpha / \alpha_{0}\right) \sim 0.002$ at $68 \%$ c.l. (see e.g. [29]).

\section{ACKNOWLEDGMENTS}

This work was supported by the PRIN-INAF grant "Astronomy probes fundamental physics," by the Italian Space Agency through the ASI contract Euclid- IC (I/031/ 10/0), by FCT (Portugal) through Grant No. PTDC/FIS/ $111725 / 2009$ and by the PHC-EGIDE/Programa PESSOA exchange grant "Probing Fundamental Physics with Planck" (FCT/1562/25/1/2012/S). The work of C. J. M. is funded by a Ciência2007 Research Contract, funded by FCT/MCTES (Portugal) and POPH/FSE (E. C.). We acknowledge the hospitality of the University of the Azores during the 2011 Azores School on Observational Cosmology, at which the key ideas behind this work were discussed.
[1] E. Komatsu et al., arXiv:1001.4538.

[2] J. Dunkley et al., arXiv:1009.0866.

[3] C. L. Reichardt et al., Astrophys. J. 694, 1200 (2009).

[4] R. Keisler et al., arXiv:1105.3182.

[5] B. A. Reid et al., Mon. Not. R. Astron. Soc. 404, L60 (2010).

[6] E. W. Kolb and M. S. Turner, Front. Phys. 69, 1 (1990).

[7] G. Mangano, G. Miele, S. Pastor, T. Pinto, O. Pisanti, and P. D. Serpico, Nucl. Phys. B 729, 221 (2005).

[8] M. Archidiacono, E. Calabrese, and A. Melchiorri, Phys. Rev. D 84, 123008 (2011).

[9] Z. Hou, R. Keisler, L. Knox, M. Millea, and C. Reichardt, arXiv:1104.2333.

[10] T. L. Smith, S. Das, and O. Zahn, Phys. Rev. D 85, 023001 (2012)arXiv:1105.3246.

[11] J. Hamann, arXiv:1110.4271.

[12] E. Giusarma, M. Corsi, M. Archidiacono, R. de Putter, A. Melchiorri, O. Mena, and S. Pandolfi, Phys. Rev. D 83, 115023 (2011).

[13] E. Giusarma, M. Archidiacono, R. de Putter, A. Melchiorri, and O. Mena, arXiv:1112.4661.

[14] A. Smith, M. Archidiacono, A. Cooray, F. De Bernardis, A. Melchiorri, and J. Smidt, arXiv:1112.3006.

[15] S. Joudaki, arXiv:1202.0005.

[16] S. Galli, F. Iocco, G. Bertone, and A. Melchiorri, Phys. Rev. D 84, 027302 (2011); 80, 023505 (2009).

[17] R. A. Battye, R. Crittenden, and J. Weller, Phys. Rev. D 63, 043505 (2001).

[18] P. P. Avelino et al., Phys. Rev. D 64, 103505 (2001); C. J. A. Martins, A. Melchiorri, R. Trotta, R. Bean, G. Rocha, P. P. Avelino, and P. T. P. Viana, Phys. Rev. D 66, 023505 (2002).
[19] C. J. A. Martins, A. Melchiorri, G. Rocha, R. Trotta, P. P. Avelino, and P. T. P. Viana, Phys. Lett. B 585, 29 (2004); G. Rocha, R. Trotta, C. J. A. Martins, A. Melchiorri, P. P. Avelino, R. Bean, and P. T. P. Viana, Mon. Not. R. Astron. Soc. 352, 20 (2004).

[20] K. Ichikawa, T. Kanzaki, and M. Kawasaki, Phys. Rev. D 74, 023515 (2006).

[21] P. Stefanescu, New Astron. Rev. 12, 635 (2007).

[22] M. Nakashima, R. Nagata, and J. Yokoyama, Prog. Theor. Phys. 120, 1207 (2008).

[23] E. Menegoni, S. Galli, J. G. Bartlett, C. J. A. Martins, and A. Melchiorri, Phys. Rev. D 80, 087302 (2009); E. Menegoni et al., Int. J. Mod. Phys. D 19, 507 (2010); E. Calabrese, E. Menegoni, C. J. A. P. Martins, A. Melchiorri, and G. Rocha, Phys. Rev. D 84, 023518 (2011).

[24] C. G. Scoccola, S. J. Landau, and H. Vucetich, Phys. Lett. B 669, 212 (2008); S. J. Landau and C. G. Scoccola, arXiv:1002.1603.

[25] J. K. Webb et al., Phys. Rev. Lett. 107, 191101 (2011).

[26] A. Lewis and S. Bridle, Phys. Rev. D 66, 103511 (2002); Available from http://cosmologist.info.

[27] A. G. Riess, L. Macri, S. Casertano, H. Lampeitl, H. C. Ferguson, A. V. Filippenko, S. W. Jha, W. Li et al., Astrophys. J. 730, 119 (2011).

[28] H. Trac, P. Bode, and J. P. Ostriker, Astrophys. J. 727, 94 (2011).

[29] S. Galli, M. Martinelli, A. Melchiorri, L. Pagano, B. D. Sherwin, and D. N. Spergel, Phys. Rev. D 82, 123504 (2010); M. Shimon, N. J. Miller, C. T. Kishimoto, C. J. Smith, G. M. Fuller, and B. G. Keating, J. Cosmol. Astropart. Phys. 05 (2010) 037. 